\title{
Genetic control of anthocyanin pigmentation of potato tissues
}

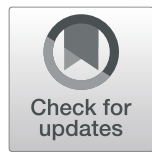

\author{
Ksenia V. Strygina ${ }^{1,3^{*}}$, Alex V. Kochetov ${ }^{1,2}$ and Elena K. Khlestkina ${ }^{1,2,3}$ \\ From 11th International Multiconference "Bioinformatics of Genome Regulation and StructurelSystems Biology" - BGRSISB- \\ 2018
}

Novosibirsk, Russia. 20-25 August 2018

\begin{abstract}
Background: The cultivated potato Solanum tuberosum L. is the fourth most important crop worldwide. Anthocyanins synthesis and accumulation in potato tissues are considered as one of important traits related to stress resistance and nutritional value. It is considered that the major regulatory gene for anthocyanin biosynthesis is R2R3 MYB-encoding gene StAN1. However, the genetic control of pigmentation of different potato tissues is substantially under investigated. The development of genetic markers for breeding of potato with specific pigmentation pattern remains an actual task.

Results: We investigated 36 potato varieties and hybrids with different pigmentation of tubers and leaves. Sequence organization of regulatory R2R3 MYB (StAN1, StMYBA1, StMYB113), bHLH (StbHLH1, StJAF13) and WD40 (StWD40) genes potentially controlling anthocyanin biosynthesis has been evaluated. The results demonstrated a high variability in the StAN1 third exon and promoter region with the exception for $35 \mathrm{bp}$, containing elements for the transcription start and activation of gene expression in roots. The analysis of transcriptional activity of genes coding R2R3 MYBs, bHLHs and WD40 transcriptional factors in leaves of eight potato genotypes with different anthocyanin pigmentation was performed. The results showed a relation between the gene expression level and plant pigmentation only for the StAN1 and StWD40 genes, while other studied genes had either strong expression in all varieties and hybrids (STMYBA1, StbHLH1 and StJAF13) or they were not expressed at all (STMYB113).

Conclusions: It was found that StAN1 is the major regulatory gene controlling potato anthocyanin synthesis. However, diagnostic markers developed for the functional StAN1 alleles $\left(\operatorname{StAN} 1^{777}\right.$ and StAN1 ${ }^{816}$ ) can not be used efficiently for prediction of potato pigmentation patterns. It is likely that the sequence organization of StAN1 promoter is important for anthocyanin synthesis control and the development of additional diagnostic markers is necessary.
\end{abstract}

Keywords: Anthocyanins, Diagnostic markers, Gene transcription, Solanum tuberosum, Marker-assisted selection

\section{Background}

Potato Solanum tuberosum L. is an important food crop. Potato tubers contain a significant amount of polyphenols, including water-soluble pigments anthocyanins, which can occur in some genotypes [1]. Anthocyanins are widely distributed in the plant kingdom. These

\footnotetext{
* Correspondence: pushpandzhali@bionet.nsc.ru

'Institute of Cytology and Genetics, Siberian Branch of the Russian Academy of Sciences, Lavrentjeva Ave. 10, Novosibirsk 630090, Russia

${ }^{3}$ N.I. Vavilov All-Russian Research Institute of Plant Genetic Resources (VIR),

Bolshaya Morskaya Str., 42-44, St. Petersburg 190000, Russia

Full list of author information is available at the end of the article
}

pigments colourize the vegetative and generative plant organs [1]. Anthocyanins presenting in fruits and flowers provide visual signals for attraction of pollinators and seed dispersers [2,3]. Besides, anthocyanins also protect plants against various biotic and abiotic stresses due to their antioxidant properties and make possible nutritional and medicinal contribution to human health [1, 4]. It was shown that the pigmented potato genotypes (especially with red and purple skin and flesh) have significantly higher antioxidant activity [5]. Thus, potatoes with a high content of anthocyanins are important in

(C) The Author(s). 2019 Open Access This article is distributed under the terms of the Creative Commons Attribution 4.0 International License (http://creativecommons.org/licenses/by/4.0/), which permits unrestricted use, distribution, and 
terms of health. From the other side, colouration of the stem and leaves as adaptive features are noteworthy [6].

A number of genes described previously regulate the biosynthesis of anthocyanins. It is considered that the major regulators are the genes encoding the transcription factors R2R3 MYB, bHLH (basic helix-loop-helix) and WD40 forming the MBW complex (MYB-bHLH-WD40) [7-9]. There were several R2R3 MYB-encoding genes considered to be the regulators of phenylpropanoid biosynthesis pathway, among them StAN1 (ANTHOCYANIN1) was the main candidate gene for regulation synthesis of anthocyanins in potato tubers and leaves [10-13]. It has been suggested that the presence of one or two r-repeats (perfect $30 \mathrm{bp}$ long duplication coding for 10 amino acids TIAPQPQEGI; alleles StAN1-r1 and StAN1-r2, respectively) in the third exon of the StAN1 gene is crucial for its proper regulatory functioning (Fig. 1) [13, 14]. Besides, the expression of StAN1 correlated with the level of flavonoids in drought-stressed potatoes [15]. Expression of the highly homologous gene StMYBA1 / StAN2 was noted in tubers of pigmented and uncoloured genotypes [13]. Functional studies have shown that StMYBA1 is less able to induce anthocyanins than StAN1, but it has a strong ability to induce production of monolignol and hydroxycinnamic acid derivatives [16, 17]. It has been shown that in a cold-resistant wild potato $S$. commersonii this gene is capable of responding to cold stress [16]. The R2R3 MYB-encoding gene StMYB113 (homologous to the Arabidopsis gene AtMYB113, which positively regulates the metabolism of phenylpropanoids) also demonstrated transcriptional activity in potato tubers [13]. The bHLH-encoding genes StbHLH1 and StJAF13 show a correlation with the expression of phenylpropanoid genes in potato leaves and tubers [11-13]. It has been revealed that
StJAF13 regulates the expression of StAN1, which then activates the structural genes $C H I, F 3 H, F 3 ' H$ and $A N S$ of tobacco plant [18]. In addition, it has been shown that the expression level of WD40-coding gene StWD40 correlates with total phenolics and anthocyanins content in the red and purple potato tubers [11]. Further investigation of the regulatory genes related to anthocyanin biosynthesis in potato, and development of DNA markers diagnostic for their dominant and recessive alleles would provide the base for the accelerated breeding of potato with desired skin and flash colour.

Current study is aimed on (i) characterization of the StAN1 gene and development of DNA markers diagnostic for the functional alleles of this gene as well as (ii) investigation of the functional allelic variability of the known R2R3 MYB-, bHLH- and WD40-coding genes among eight potato genotypes differing in anthocyanin pigmentation.

\section{Results \\ StAN1 allelic variability \\ Third exon}

High variability in the StAN1 genomic sequence was previously identified in the 3rd exon [12-14]. The ability to regulate the anthocyanins biosynthesis in potato is provided by the presence in StAN1 of one or two perfect r-repeats (Fig. 1). We developed DNA marker based on StAN1 alleles inclusive from zero to three repeats (Table 1). This marker flanks all $\mathrm{r}$-repeats in the third exon of StAN1 gene. Thus, it is possible to select the functional alleles of $S t A N 1$ by the amplicon length: StAN1-r0-296 bp, StAN1-r1-338 bp, StAN1-r2-377 bp, StAN1-r3-398 bp. The marker suitability was confirmed by sequencing of the corresponding PCR products. With

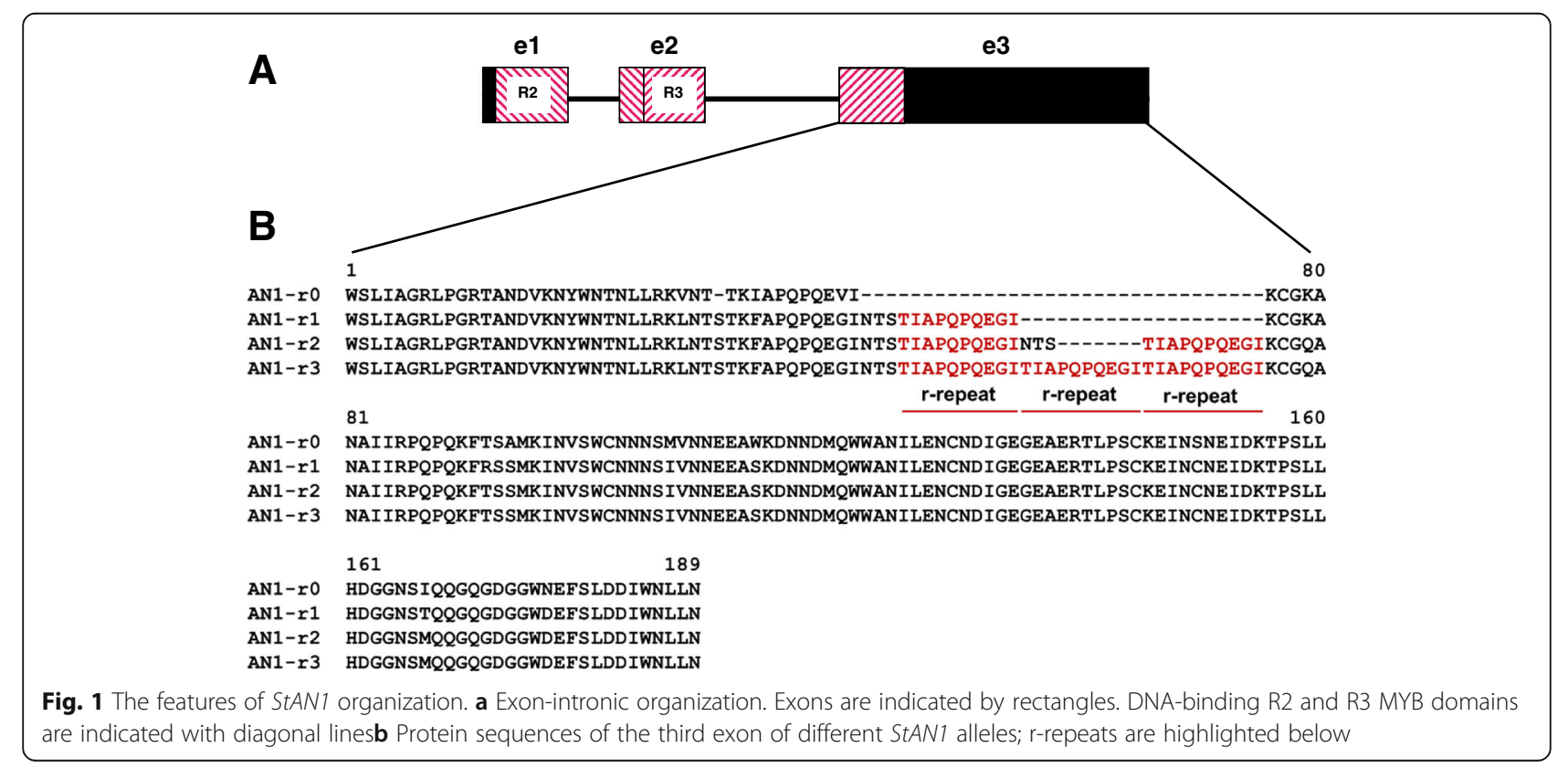


Table 1 Gene-specific primers used in current study

\begin{tabular}{|c|c|c|c|c|c|c|c|}
\hline \multirow[t]{2}{*}{ Gene } & \multirow[t]{2}{*}{ GenBank ID } & \multirow[t]{2}{*}{ Purpose } & \multirow[t]{2}{*}{$\begin{array}{l}\text { Annealing } \\
\text { temperature }\left({ }^{\circ} \mathrm{C}\right)\end{array}$} & \multirow[t]{2}{*}{$\begin{array}{l}\text { Forward primer (FP, } 5^{\prime} \rightarrow \\
\left.3^{\prime}\right)\end{array}$} & \multirow[t]{2}{*}{ Reverse primer (RP, $\left.5^{\prime} \rightarrow 3^{\prime}\right)$} & \multicolumn{2}{|c|}{$\begin{array}{l}\text { Primer binding } \\
\text { site }\end{array}$} \\
\hline & & & & & & $\overline{F P}$ & $\mathrm{RP}$ \\
\hline \multirow[t]{5}{*}{ StAN1 } & $\begin{array}{l}\text { KM822778 } \\
\text { AY841130 } \\
\text { AY8411128 } \\
\text { KM822780 }\end{array}$ & diagnostic PCR, sequencing & 55 & GGAAGGACAGCTAACGATG & AGTGTTCTTTCAGCTTCTCC & E3 & E3 \\
\hline & $\begin{array}{l}\text { chr10:51745 } \\
\text { 200-51,749,200 }\end{array}$ & $P C R$, sequencing & 50 & GTCACATCACTACACCACAT & $\begin{array}{l}\text { TCCACTTCATCCCAATCAA } \\
\text { AG }\end{array}$ & promoter & E2 \\
\hline & AY841128 & $\begin{array}{l}\text { full length CDS amplification, } \\
\text { sequencing }\end{array}$ & 50 & $\begin{array}{l}\text { ATGACTTCACATGTAATGA } \\
\text { TCAT }\end{array}$ & $\begin{array}{l}\text { CTAATTAAGTAGATTCCAT } \\
\text { ATATCA }\end{array}$ & E1 & E3 \\
\hline & AY841128 & qRT-PCR (a) & 60 & $\begin{array}{l}\text { GGAGAAGGAAAGTGGCATC } \\
\text { TTGTTCCA }\end{array}$ & $\begin{array}{l}\text { TCCACTTCATCCCAATCAA } \\
\text { AG }\end{array}$ & E1 & E2 \\
\hline & AY841128 & qRT-PCR (b) & 60 & $\begin{array}{l}\text { GAGAAGCTGAAAGAACACT } \\
\text { ACCT }\end{array}$ & CACCATCACCTTGTCCTTGT & E3 & E3 \\
\hline StMYBA1 & JQ219855 & qRT-PCR & 60 & $\begin{array}{l}\text { GTGGTCACTTATTGCTGGT } \\
\text { AGA }\end{array}$ & $\begin{array}{l}\text { GGCGAGGAGGAGGAGTA } \\
\text { ATA }\end{array}$ & E3 & E3 \\
\hline StMYB113 & KU242748 & qRT-PCR & 60 & $\begin{array}{l}\text { CTAGGCAACAGATGGTCAC } \\
\text { TTAT }\end{array}$ & $\begin{array}{l}\text { CTTCCTGTGTAGGTGTGTG } \\
\pi\end{array}$ & E3 & E3 \\
\hline StbHLH1 & JX848660 & qRT-PCR & 60 & $\begin{array}{l}\text { CCACCAAAGCCAGCTTTAT } \\
\text { C }\end{array}$ & $\begin{array}{l}\text { ATCCGCTGGACAAATACC } \\
\text { AG }\end{array}$ & E5 & E5 \\
\hline StJAF13 & KP317176 & qRT-PCR & 60 & $\begin{array}{l}\text { CTGCAGAGCAGACATCTGA } \\
\text { TAA }\end{array}$ & $\begin{array}{l}\text { GCAGCTTTCAGGTTCCATT } \\
\text { TC }\end{array}$ & E7 & E7 \\
\hline StWD40 & JX848661 & qRT-PCR & 60 & $\begin{array}{l}\text { ACCCTTAAGCCTGTTCCAA } \\
\text { ATC }\end{array}$ & $\begin{array}{l}\text { CACCGGAAGAGGCAAG } \\
\text { AATATC }\end{array}$ & E1 & E1 \\
\hline
\end{tabular}

these markers, 36 DNA samples of potato varieties and hybrids with different anthocyanins pigmentation of tubers skin (no/pink/red/purple), tuber flash (no/purple) and leaves (no/purple) were analysed (Table 2, Fig. 2a, Additional file 1). Functional alleles StAN1-r1 and StAN1-r2 were identified in 36 and 18 samples, respectively. All samples with the exception of Zlatka and 1013/ 3-1 have the non-functional allele StAN1-rO. In five samples, StAN1-r3 allele was found (Aroza, Favorit, 419/ 8-1, 710/10-5, 478). Thus, it was established that all the potato samples hold a functional StAN1 allele for anthocyanin biosynthesis activation.

\section{Promoter region and 1st intron}

Low conservatism was previously noted for the StAN1 gene including the promoter region. In addition to the promoter, the intron 1 often acts as a regulator of gene transcription $[19,20]$. Therefore, mutations in these regions could be critical in the transcription regulation. StAN1 promoter and 1st intron sequences of eight randomly selected potato samples and one sequence from PGSC database (chr10:51745200,51,749,200) were studied here. The $15 \mathrm{bp}$ indel mutation in the 1 st intron was found both in pigmented and unpigmented genotypes (Fig. 3). Minor variation between individual promoter sequences of potato samples were found including single nucleotide deletion and polymorphisms. However, the analysed potato samples were similar to each other in the promoter region and completely different from the database sequence except for $35 \mathrm{bp}$ (Fig. 3). The analysis with the New PLACE database revealed that the conservative region of StAN1 promoter sequences share light-responsive Inr (initiator) element with signal sequence YTCANTYY which is necessary for promoters without TATA boxes (Fig. 3, Additional file 2). In addition, all promoter sequences have a set of common elements like root-specific elements CTCTT and ATAT $\mathrm{T}$, tetranucleotide CACT of mesophyll expression module and CAAT-boxes (promoter consensus sequence) (Additional file 2).

\section{Expression analysis of regulatory $R 2 R 3 M Y B, b H L H$ and WD40 genes}

Among 36 potato varieties and hybrids, eight samples characterized by marked colour differences of leaves, tuber flesh and skin were selected: Safo, Lina, Fioletovy, Koldovskaya, 710/10-5, 1014/9-1, 790/1-5, 418/3 (Fig. $2 \mathrm{~b}$, Table 2). From the leaves of these plants, RNA preparations were isolated to assess the expression levels of the R2R3 MYB, bHLH and WD40 genes involved in anthocyanin biosynthesis control.

\section{Analysis of StAN1, StMYBA1 and StMYB113 in differentially pigmented potato leaves}

To investigate the expression profiles of R2R3 MYB gene StAN1 in potato leaves, two primer pairs were designed 
Table 2 Genetic stocks of potato samples and their phenotypic characteristics that were used to characterize StAN1 alleles. Cultivars and hybrids used for qPCR amplification are underlined

\begin{tabular}{|c|c|c|c|c|c|c|}
\hline \multirow[t]{2}{*}{ № } & \multirow{2}{*}{$\begin{array}{l}\text { Cultivars and } \\
\text { hybrids designation }\end{array}$} & \multirow{2}{*}{$\begin{array}{l}\text { Presence of leaf and } \\
\text { stem anthocyanin } \\
\text { coloration }\end{array}$} & \multicolumn{2}{|c|}{ Tuber coloration } & \multirow[t]{2}{*}{ Pedigree of the hybrids } & \multirow{2}{*}{$\begin{array}{l}\text { StAN1 } \\
\text { alleles }\end{array}$} \\
\hline & & & Tuber flesh & Tuber skin & & \\
\hline 1 & Zlatka & - & yellow & yellow with redbuds & & $r 1, r 2$ \\
\hline 2 & Yuna & - & yellow & pink & & $r 0, r 1$ \\
\hline 3 & Safo & - & white & white & & $r 0, r 1$ \\
\hline 4 & Lina & + & yellow & yellow & & $r 0, r 1$ \\
\hline 5 & $710 / 10-5$ & + & yellow & red with whitebuds & Symphonia x Adretta & $r 0, r 1, r 3$ \\
\hline 6 & $1-7-5 A$ & - & yellow & red & Zhukovsky ranniy $x$ Ute & $r 0, r 1, r 2$ \\
\hline 7 & $1-9-2$ & - & white & white & 1409-4/86 x Rossiyanka & $r 0, r 1$ \\
\hline 8 & $2-5-4 B$ & - & yellow & red & 244-1 x Karlena & $r 0, r 1, r 2$ \\
\hline 9 & $1-14-2 \mathrm{~A}$ & - & yellow & white with pinkbuds & Helena $\times$ 946-3 & $r 0, r 1, r 2$ \\
\hline 10 & $1014 / 3-1$ & - & white & white with pinkbuds & Nikulinsky x Picasso & $r 0, r 1, r 2$ \\
\hline 11 & $821 / 1-5$ & - & yellow & white with pinkbuds & Nikulinsky x Picasso & $r 0, r 1, r 2$ \\
\hline 12 & $419 / 8-1$ & - & yellow & pink & Zhukovsky ranniy x CM №1 & $r 0, r 1, r 3$ \\
\hline 13 & $1014 / 8-1$ & + & cream & white with pinkbuds & Nikulinsky x Picasso & $r 0, r 1, r 2$ \\
\hline 14 & $1013 / 3-1$ & - & white & white with pinkbuds & Nikulinsky x Picasso & $r 0, r 1$ \\
\hline 15 & $790 / 1-5$ & - & yellow & red & Nikulinsky x Omega & $r 0, r 1, r 2$ \\
\hline 16 & $597 / 4-1$ & - & yellow & red & Zhukovsky ranniy x CM №1 & $r 0, r 1, r 2$ \\
\hline 17 & $999 / 1-1$ & - & cream & white & Sentyabr x Latona & $r 0, r 1, r 2$ \\
\hline 18 & $785 / 8-5$ & - & yellow & red & Symphonia $\times$ Pushkinets & $r 0, r 1, r 2$ \\
\hline 19 & $2-5-2$ & - & yellow & yellow & 244-1 x Karlena & $r 0, r 1$ \\
\hline 20 & $826 / 1-5$ & - & white & white with pink buds & Granola × 234-90 & $r 0, r 1, r 2$ \\
\hline 21 & Favorit & + & cream & pink & & $r 0, r 1, r 3$ \\
\hline 22 & Liubava & + & white & red & & $r 0, r 1$ \\
\hline 23 & Fioletovy & + & purple & purple & & $r 0, r 1$ \\
\hline 24 & Koldovskaya & + & purple & purple & & $r 0, r 1$ \\
\hline 25 & Nakra & + & yellow & red & & $r 0, r 1, r 2$ \\
\hline 26 & Meteor & + & yellow & yellow & & $r 0, r 1$ \\
\hline 27 & Kuznechanka & + & cream & red & & $r 0, r 1, r 2$ \\
\hline 28 & Aroza & + & yellow & red & & $r 0, r 1, r 3$ \\
\hline 29 & Fritella & + & white & white & & $r 0, r 1$ \\
\hline 30 & $418 / 3$ & + & white and red & purple & Picasso x Adretta & $r 0, r 1$ \\
\hline 31 & $479 / 5$ & + & yellow & yellow & Ausonia x Naiada & $r 0, r 1$ \\
\hline 32 & $417 / 2$ & + & yellow & red & 1-11-5 x Pamyati Osipovoy & $r 0, r 1, r 2$ \\
\hline 33 & Bekas & + & white & white & & $r 0, r 1$ \\
\hline 34 & Fregata & + & white & white & & $r 0, r 1, r 2$ \\
\hline 35 & 478 & + & yellow & red & Irbitsky x Naiada & $r 0, r 1, r 3$ \\
\hline 36 & $1753 / 6$ & + & yellow & purple & BP $808 \times 88.34 / 14$ & $r 0, r 1$ \\
\hline
\end{tabular}

for different gene regions (Table 1, Additional file 3). It was found that different primer pairs ( $\mathbf{a}$ and $\mathbf{b})$, used in qPCR analysis, give different expression results (Fig. 4). StAN1 expression in the R2R3 domain region (primers combination a) was higher in genotypes without or with weak leaf anthocyanin pigmentation than in coloured genotypes Koldovskaya and Fioletovy (Fig. 4). On the other hand, the expression of this gene in the third exon region (primers combination $\mathbf{b}$ ) is associated with leaves pigmentation. We assume that the second primer pair (b) was more specific to detect functional StAN1 alleles. An exception was genotype $790 / 1-5$, in which we 


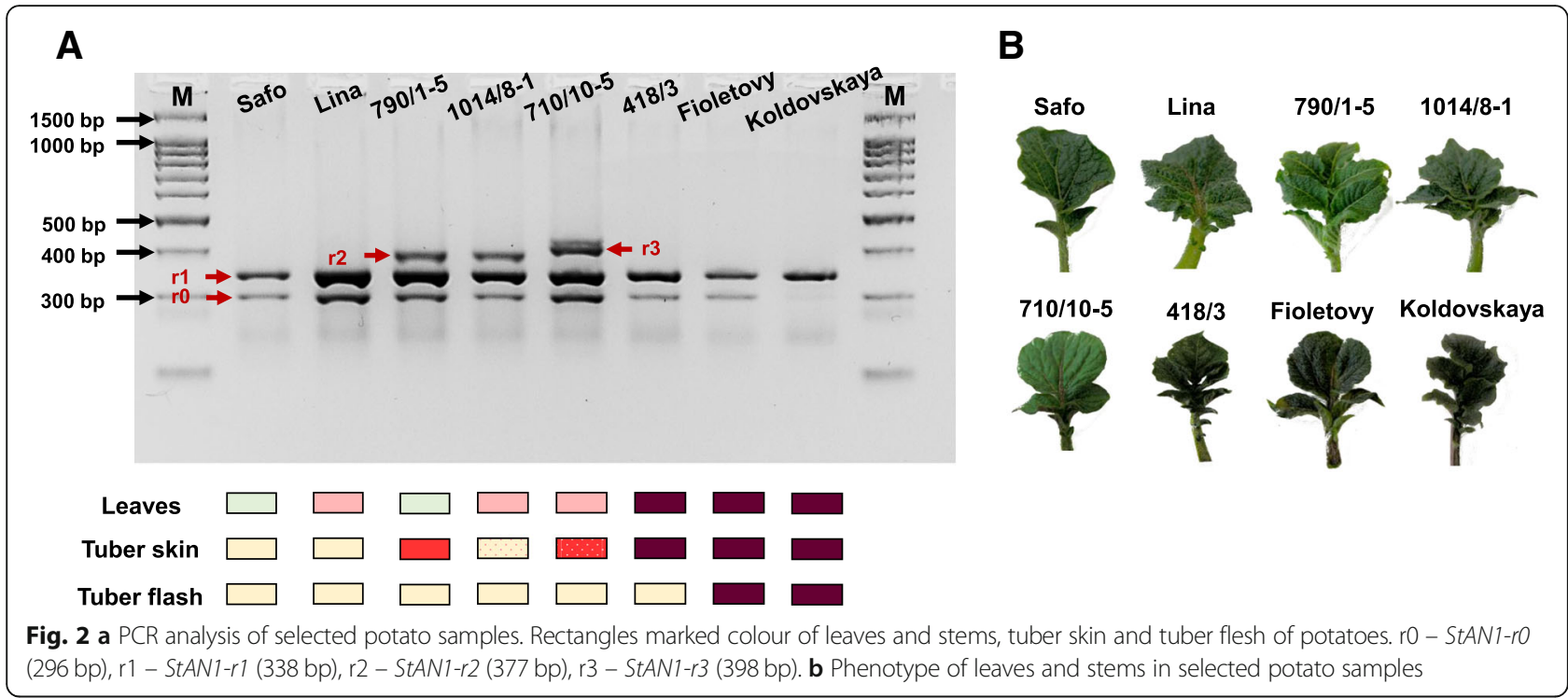

detected high level of StAN1 expression despite the absence of leave anthocyanin pigmentation (Fig. 4). Full-length coding sequence of only StAN1-r2 allelic variant was amplified from cDNA of leaves of 790/1-5 hybrid. For comparison, only one StAN1 allelic variant named StAN1-r1 was also amplified for genotype Fioletovy with strong anthocyanin pigmentation (GenBank: MH796171-MH796172).

The relative expression levels of the R2R3 MYB-coding genes StMYBA1 and StMYB113 were also analysed. We examined the transcriptional activity of StMYB113. Its mRNA was not detected in the leaves of any genotype. Expression of StMYBA1 was detected at a high level in the analysed tissue (Fig. 4), but there was no relationship between relative level of gene expression and the presence of anthocyanins in potato leaves.

\section{qPCR analysis of StbHLH1, StJAF13 and StWD40 in differentially pigmented potato leaves}

Expression levels of two bHLH (StbHLH1 and StJAF13) and one WD40 (StWD40) transcription factor genes was studied. The transcription of StbHLH1 was detected all analysed genotypes (Fig. 4). However, the strongest expression level was detected in uncoloured cultivar Safo and in hybrid 710/10-5 with weak leave pigmentation. StJAF13 gene demonstrated the transcriptional activity at

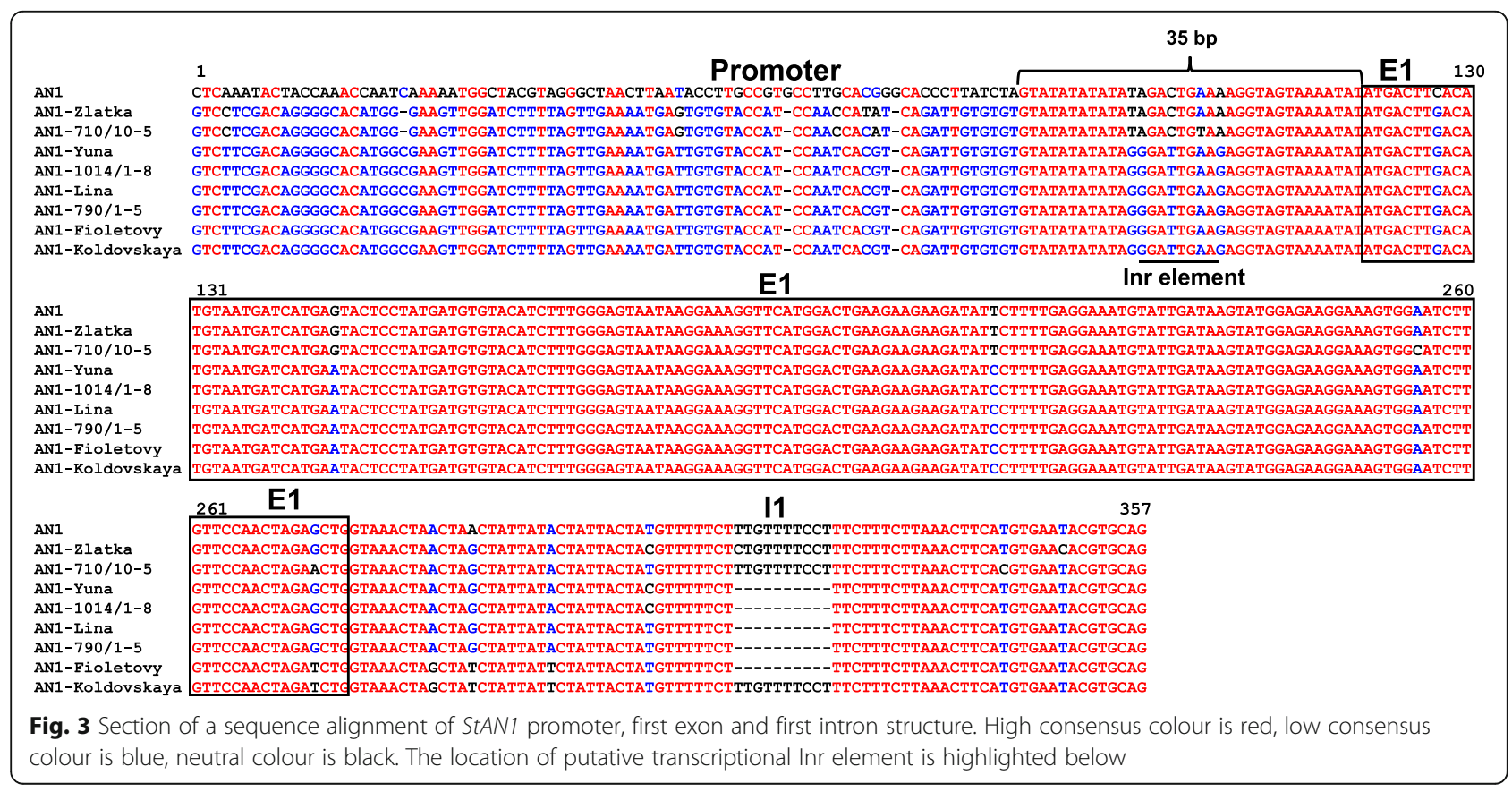




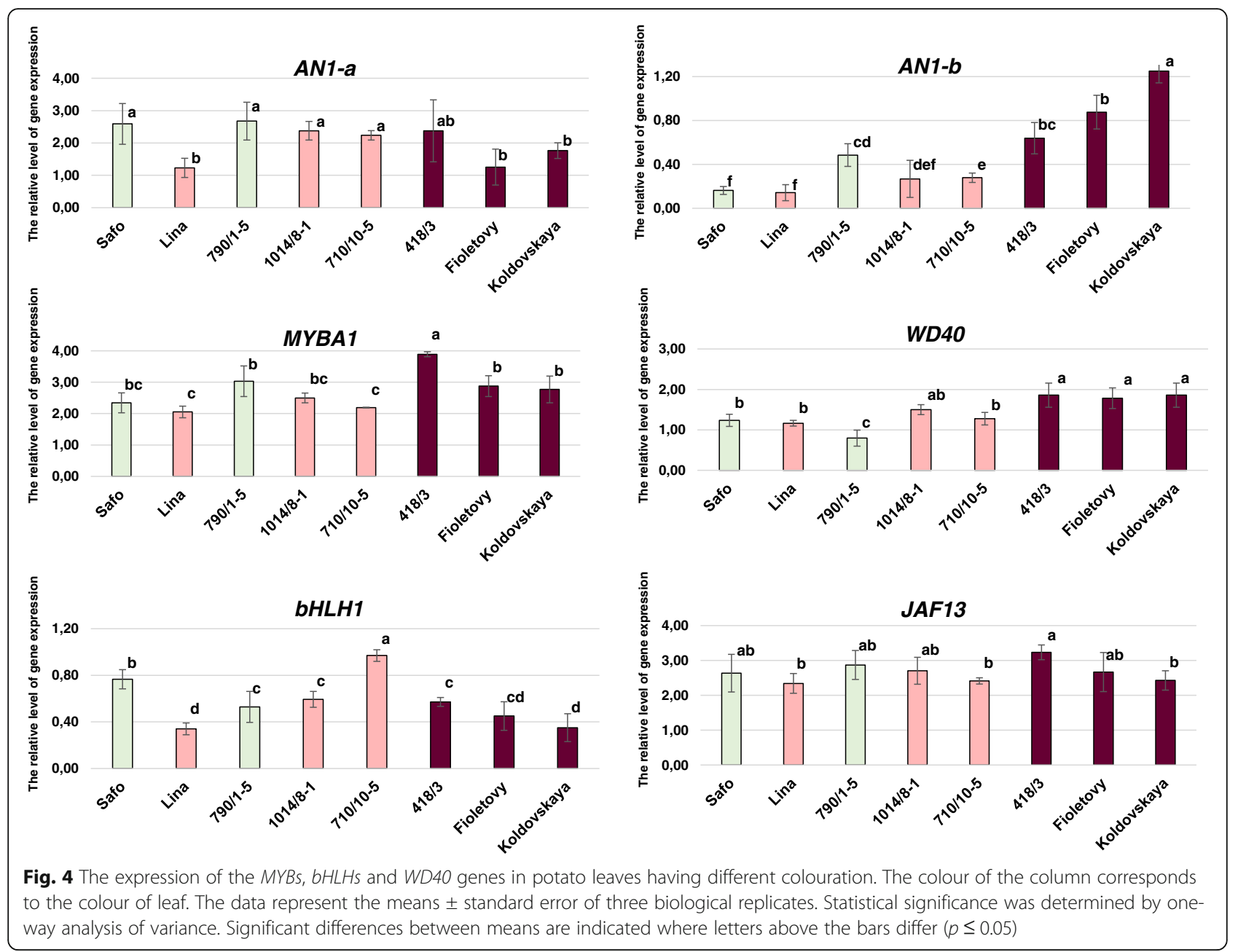

approximately the same high level in all genotypes, regardless of their pigmentation (Fig. 4). From the other side, it was shown that the strong expression level of the StWD40 gene was associated with the anthocyanin colouration: in genotypes 418/3, Koldovskaya and Fioletovy with strong anthocyanin coloration relative expression level was significantly higher than in unpigmented genotypes Safo and 790/1-5 (Fig. 4).

\section{Discussion}

Plant pigments anthocyanins belong to a large group of plant phenolic compounds called flavonoids. Many studies have shown health benefits with a significant amount of regularly consumed anthocyanins, which potentially helps in preventing various chronic pathologies [1]. However, most plants contain only small amounts of anthocyanins in edible parts $[4,21]$. Epidermis coloured with anthocyanins and uncoloured flesh, consisting for example of white parenchymal cells, is the most common pigmentation of plant organs. This pigmentation occurs in purple cabbage, tomatoes, onions, eggplants and grapes. Nevertheless, there are examples of plants with edible organs with parenchyma coloured with anthocyanins, such as S. tuberosum with purple flesh [22].

The anthocyanin biosynthetic genes are transcriptionally regulated by ternary $\mathrm{MBW}$ protein complex containing R2R3 MYB, bHLH and WD40 transcription factors [7-9]. To date, there are three R2R3 MYB-encoding genes potentially involved in the biosynthesis of anthocyanins in the potato genome: StAN1, StMYBA1 and StMYB113. Additionally, two bHLH genes (StJAF13 and StbHLH1) and one WD40 (StWD40) gene have been revealed in S. tuberosum genome. The genes StAN1, StbHLH1 and StJAF13 have been identified as positive regulators for pigmentation of potato leaves and tuber skin and flesh $[12,13]$. In contrast to conservative bHLH- and WD40-encoding genes, the MYB-encoding StAN1 gene is variable [14].

\section{Major variants of StAN1 present in potato cultivars and hybrids}

Previously, it was shown that for the proper functioning StAN1 should contain one or two perfect r-repeats in its 
3rd exon (Fig. 1). These alleles were designated as StAN1-r1 and StAN1-r2, respectively (formerly $S t A N 1^{777}$ and $\left.S t A N 1^{816}[10]\right)$. It was found that in all potato samples that we analysed the functional StAN1-r1 and StAN1-r2 alleles are contained (Fig. 2a, Additional file 1).

Although the structure of the 3rd exon of the gene plays the key role in the anthocyanins biosynthesis [14], it appeared to be not the only one critical element - the differences between allelic variants should also be found in the promoter region. However, our results demonstrated a high variability in the StAN1 promoter with the exception of $35 \mathrm{bp}$, containing elements of the transcription start and activation of gene expression in the plant roots (Fig. 3, Additional file 2). Apparently, some certain cis-regulatory enhancer element can determine the activity of the gene. Perhaps this is a putative SINE (short interspersed elements, (TA) ${ }_{36}$ repeat at $-1969 \mathrm{bp}$ from the ATG site) element detected in D'Amelia et al. [12]. The presence of a putative SINE retrotransposon in StAN1 promoter may explain differences between green leaf samples and leaves with abundant anthocyanin pigmentation. A similar pattern was observed for the bHLH-like transcription factor TaMyc-A1 in Triticum aestivum genome, which is involved in the anthocyanins biosynthesis in wheat pericarp [23, 24]. The difference between TaMyc-A1 alleles are in variation of number of the 261 bp-element upstream the transcription start site. Tandem duplication of this element has influence on activation of TaMyc-A1 expression and appearance of abundant anthocyanin coloration in wheat pericarp. Thus, for the selection of potato varieties with anthocyanin pigmentation it is necessary to develop additional PCR markers flanking putative SINE element.

\section{StAN1 and StWD40 regulate anthocyanins synthesis in leaf} It was detected that within the structural part of the StAN1 gene, the first exon is the most conserved region (Fig. 3). To analyse the relative expression level of the StAN1 gene, we developed primers to this region containing necessary R2R3 motif (combination a) and to the 3rd exon with perfect $r$-repeats (combination b) (Table 1, Additional file 3). Our analysis revealed that the primer pair $\mathbf{b}$ was proved to be more suitable to detect functional StAN1 alleles (Fig. 4). A similar difference has been observed in Liu et al. [13] in the analysis of StAN1 expression in potato tubers. It was found that the truncated version of StAN1-r0 (StAN1-rOT) was amplified from cDNA of white skinned potato sample at positions 1-302 bp. The truncated version of StAN1 did not promote anthocyanin synthesis at any level or significantly inhibit the activity of full-length StAN1. Thus, we assumed that major transcription variants of StAN1 amplified from cDNA of uncoloured or weakly pigmented genotypes are truncated.
The independent colour of potato leaves and tubers is determined by the functional MBW complex, wherein the MYB component is the product of StAN1 gene, but bHLH and WD40 components could be different [13, 14]. Indeed, a comparative analysis of StAN1 expression with primers combination $\mathbf{b}$ revealed a relationship between the intensity of the anthocyanin colour of the plant and the level of its expression (Fig. 4). Among the samples analysed StAN1 expression was not associated with anthocyanin pigment only in 790/1-5 hybrid sample. Due to the presence of red anthocyanin coloration in skin of hybrid tuber, we assumed that the lack of pigments in the leaves is related to the mutations in a co-regulator gene (presumably bHLH), controlling pigmentation in the leaves, but not in the tubers.

The relationship between the transcription level and the phenotype of the potato was also observed for the StWD40 gene. Despite the fact that the level of its expression was high in plants of all the potato genotypes tested, the tendency of increased expression in pigmented samples and decreased expression in green ones was noted (Fig. 4). Expression patterns of StWD40 suggest that it is an important factor determining anthocyanins amount in potato leaf. Previously, the expression of this gene was not detected in leaves, but a similar pattern was observed in potato tubers.

The relationship between the expression of other regulatory genes and the phenotype of the analysed tissue was not revealed (Fig. 4). It was established that StMYBA1 is expressed in all analysed genotypes. Previously it was shown that in a cold-resistant wild potato $S$. commersonii an ortholog of this gene activated production of monolignol and hydroxycinnamic acid derivatives, which probably may be related with cold tolerance [16]. It is possible that in S. tuberosum the function of StMYBA1 was lost during the evolution and domestication. StMYB113 demonstrated complete absence of expression in the leaves of the potato. Previous study demonstrated the presence of StMYB113 transcripts in potato tubers regardless of their pigmentation [13]. Thus, the gene StMYB113 has a tissue-specific expression.

Previously, the relative levels of expression of both bHLH genes (StJAF13 and StbHLH1) in the leaves were measured only in the paper of D'Amelia and co-authors: StbHLH1 transcription showed no association with colour, while for StJAF13 association was found [12]. In our research expression of StJAF13 and StbHLH1 in Russian potato samples was not associated with the leaf phenotype. However, we demonstrated that StJAF13 has a conservative expression profile, even in the absence of pigmentation. These data revealed that the most important regulator controlling potato anthocyanin synthesis is StAN1. This gene should be targeted for further marker-assisted selection. 


\section{Conclusions}

It was found that anthocyanin synthesis in potato leaves is mainly controlled by regulatory StAN1 gene. The structural organization of StAN1 gene in different potato cultivars was determined. The intragenic diagnostic marker for detection of different StAN1 alleles according to the number of $\mathrm{r}$-motifs was developed. For the first time it was demonstrated that the expression of another transcription factor StWD40 is also related to the colour of the potato leaves. These results provided new information on genetic control of potato pigmentation and may also be useful for further development of diagnostic marker for potato breeding.

\section{Methods}

\section{Plant materials}

Plant material were selected from GenAgro genetic collection and includes 36 varieties and hybrids of the Russian tetraploid potato $S$. tuberosum (Table 2). These potato samples were screened for the presence of the StAN1 allelic variants (Table 2). Eight samples contrasting in anthocyanin pigmentation were selected for analysis of the expression of anthocyanin biosynthesis genes (Table 2, grey colour). The plants were grown in greenhouse facility of Institute of Cytology and Genetics SB RAS under a $14 \mathrm{~h}$ photoperiod.

\section{DNA and RNA extraction, cDNA synthesis}

Total genomic DNA was extracted from leaf material applying a DNeasy Plant Mini Kit (QIAGEN, Germany). For total RNA extraction from fresh potato leaves a Plant RNA Mini Prep ${ }^{\mathrm{TM}}$ kit (Zymo Research Corporation, USA) was used. Three biological replicates were prepared for each genotype. All isolated RNAs were treated with RNase-free DNase set (QIAGEN, Germany). Total RNA was converted to single-stranded cDNA from a template consisting of $0.8 \mu \mathrm{g}$ of total RNA using a RevertAid First Strand cDNA Synthesis Kit (Thermo Fisher Scientific Inc., USA). Testing of the effect of DNase for DNA digestive was verified by subsequent PCR with primers to the reference Actin gene (GenBank: X55749) flanking the intron (5'GATGCTCCACGAGCTGTATT3' / 5'TTCACGTCCCTGACGATTTC3').

\section{In silico analysis and primers design}

Multiple sequence alignment was carried out using MultAlin (https://multalin.toulouse.inra.fr/multalin). Promoter analysis was made with New PLACE database (https:// sogo.dna.affrc.go.jp/cgi-bin/sogo.cgi?lang=en\&pj=640\&action $=$ page $\&$ page $=$ newplace $)$. Diagnostic primers for the determination of different StAN1 alleles, as well as primers for the amplification of StAN1, StMYBA1, StMYB113, StbHLH1, StJAF13 and StWD40 transcripts, were designed using the OLIGO software.
Sequences of primers, as well as conditions for PCR, are shown in Table 1.

\section{PCR, sequencing}

Amplification was made in $20 \mu \mathrm{L}$ PCRs according to [23]. The PCR products were separated in an agarose gel (Medigen, Russia; HydraGene Co., China), coloured with ethidium bromide. We have used and utilized gels stained with ethidium bromide according safety instructions. The gel image was obtained using a Molecular Imager Gel Doc XR System (Bio-Rad Laboratories, USA) using UV light. Isolation of the PCR products from the agarose gel was performed by the QIAquick Gel Extraction Kit (QIAGEN, Germany). DNA sequencing was carried out using the SB RAS Genomics core facilities (Novosibirsk, Russia).

\section{Quantitative real-time PCR (qRT-PCR)}

qRT-PCR was performed with the primers from Table 1. The amplifications were performed in an ABI Prism 7000 Sequence Detection System (Applied Biosystems, USA). The subsequent qRT-PCR was based on a SYNTOL SYBR Green I kit (Syntol, Russia). The reference sequences used were 18S rRNA (GenBank: X67238) and b-tubulin (GenBank: 609267) (primers 5'GTGACGGGTGACGG AGAAT3' / 5'ATTTATTGTCACTACCTCCCCG3' and 5’AGCTTCTGGTGGACGTTATG3' / 5’ACCAAGTTA TCAGGACGGAAGA3', respectively). Each sample was run in three technical replications. Statistical significance was determined by Kruskal-Wallis test with Statistica (http://statsoft.ru/). Significant differences between means are indicated where letters above the bars differ $(p \leq 0.05)$.

\section{Additional files}

Additional file 1: PCR analysis of 36 samples of Russian potato varieties and hybrids. r0 - StAN1-r0 (296 bp), r1 - StAN1-r1 (338 bp), r2 - StAN1-r2 (377 bp), r3 - StAN1-r3 (398 bp). (PDF 249 kb)

Additional file 2: List of putative cis-acting regulatory elements present in the StAN1 promoter. Promoter analysis was performed using New PLACE database. (PDF $51 \mathrm{~kb}$ )

Additional file 3: Schematic arrangement of primer pairs designed for qPCR analysis of StAN1 gene. Arrows indicate primers: black - primer pair a. red - primer pair b. (PDF $67 \mathrm{~kb}$ )

Abbreviations

AN1: Anthocyanin1; MBW: MYB, bHLH, WD40

Acknowledgments

Not applicable.

Funding

Publication costs are funded by the Russian Science Foundation (№ 16-1604073). Plants were provided by GenAgro genetic collection and were cultivated in the ICG greenhouse facility (supported by ICG budget project № 0324-2018-0018). 


\section{Availability of data and materials}

The sequences obtained in the current study are available at NCBI: MH796171MH796172.

\section{About this supplement}

This article has been published as part of BMC Genetics Volume 20 supplement 1, 2019: Selected articles from BGRSISB-2018: genetics. The full contents of the supplement are available online at https:// bmcgenet.biomedcentral.com/articles/supplements/volume-20-supplement1.

\section{Authors' contributions}

KVS performed all molecular-genetic experiments, carried out in silico and statistical analysis, and participated in drafting the manuscript. AVK contributed to the interpretation of data and to revising the manuscript critically. EKK contributed to the conception and design of the study, to interpretation of data and to revising the manuscript critically. All authors read and approved the final manuscript.

\section{Ethics approval and consent to participate}

Not applicable.

\section{Consent for publication}

Not applicable.

\section{Competing interests}

The authors declare that they have no competing interests.

\section{Publisher's Note}

Springer Nature remains neutral with regard to jurisdictional claims in published maps and institutional affiliations.

\section{Author details}

${ }^{1}$ Institute of Cytology and Genetics, Siberian Branch of the Russian Academy of Sciences, Lavrentjeva Ave. 10, Novosibirsk 630090, Russia. ${ }^{2}$ Novosibirsk State University, Pirogova Str., 1, Novosibirsk 630090, Russia. ${ }^{3}$ N.I. Vavilov All-Russian Research Institute of Plant Genetic Resources (VIR), Bolshaya Morskaya Str., 42-44, St. Petersburg 190000, Russia.

\section{Published: 18 March 2019}

\section{References}

1. Khoo HE, Azlan A, Tang ST, Lim SM. Anthocyanidins and anthocyanins: coloured pigments as food, pharmaceutical ingredients, and the potential health benefits. Food Nutr Res. 2017;61(1):1361779. https://doi.org/10.1080/ 16546628.2017 .1361779$.

2. Tanaka Y, Sasaki N, Ohmiya A. Biosynthesis of plant pigments: anthocyanins, betalains and carotenoids. Plant J. 2008;54(4):733-49. https://doi.org/10. 1111/j.1365-313X.2008.03447.x.

3. Rieseberg LH, Blackman BK. Speciation genes in plants. Ann Bot Lond. 2010; 106:439-55. https://doi.org/10.1093/aob/mcq126.

4. Chaves-Silva S, dos Santos AL, Chalfun-Júnior A, Zhao J, Peres LE, Benedito $V A$. Understanding the genetic regulation of anthocyanin biosynthesis in plants-tools for breeding purple varieties of fruits and vegetables. Phytochemistry. 2018;153:11-27. https://doi.org/10.1016/j.phytochem.2018. 05.013.

5. Akyol H, Riciputi Y, Capanoglu E, Caboni MF, Verardo V. Phenolic compounds in the potato and its byproducts: an overview. Int J Mol Sci. 2016;17(6):835. https://doi.org/10.3390/ijms17060835.

6. Gould KS, Dudle DA, Neufeld HS. Why some stems are red: cauline anthocyanins shield photosystem II against high light stress. J Exp Bot. 2010; 61(10):2707-17. https://doi.org/10.1093/jxb/erq106.

7. Feller A, Machemer K, Braun EL, Grotewold E. Evolutionary and comparative analysis of MYB and bHLH plant transcription factors. Plant J. 2011;66(1):94116. https://doi.org/10.1111/j.1365-313X.2010.04459.x.

8. Gonzalez A, Zhao M, Leavitt JM, Lloyd AM. Regulation of the anthocyanin biosynthetic pathway by the TTG1/bHLH/Myb transcriptional complex in Arabidopsis seedlings. Plant J. 2008;53(5):814-27. https://doi.org/10.1111/j. 1365-313X.2007.03373.x.
9. $\mathrm{Xu} \mathrm{W}$, Dubos C, Lepiniec L. Transcriptional control of flavonoid biosynthesis by MYB-bHLH-WDR complexes. Trends Plant Sci. 2015;20(3):176-85. https:// doi.org/10.1016/j.tplants.2014.12.001.

10. Jung CS, Griffiths HM, De Jong DM, Cheng S, Bodis M, Kim TS, De Jong WS The potato developer (D) locus encodes an R2R3 MYB transcription factor that regulates expression of multiple anthocyanin structural genes in tuber skin. Theor Appl Genet. 2009;120(1):45-57. https://doi.org/10.1007/s00122009-1158-3.

11. Payyavula RS, Singh RK, Navarre DA. Transcription factors, sucrose, and sucrose metabolic genes interact to regulate potato phenylpropanoid metabolism. J Exp Bot. 2013;64(16):5115-31. https://doi.org/10.1093/jxb/ ert303.

12. D'amelia V, Aversano R, Batelli G, Caruso I, Castellano Moreno M, CastroSanz AB, Chiaiese P, Fasano C, Palomba F, Carputo D. High AN 1 variability and interaction with basic helix-loop-helix co-factors related to anthocyanin biosynthesis in potato leaves. Plant J. 2014;80(3):527-40. https://doi.org/10. 1111/tpj.12653.

13. Liu Y, Lin-Wang K, Espley RV, Wang L, Yang H, Yu B, Dare A, Varkonyi-Gasic E, Wang J, Zhang J, Wang D, Allan AC. Functional diversification of the potato R2R3 MYB anthocyanin activators AN1, MYBA1, and MYB113 and their interaction with basic helix-loop-helix cofactors. J Exp Bot. 2016;67(8): 2159-76. https://doi.org/10.1093/jxb/erw014.

14. Strygina KV, Khlestkina EK. Anthocyanins synthesis in potato (Solanum tuberosum L.): genetic markers for smart breeding. Sel'skokhozyaistvennaya Biologiya. 2017;52(1):37-49. https://doi.org/10.15389/agrobiology.2017.1. 37eng.

15. André CM, Schafleitner R, Legay S, Lefèvre I, Aliaga CAA, Nomberto G, Hoffmann L, Hausman J-F, Larondelle Y, Evers D. Gene expression changes related to the production of phenolic compounds in potato tubers grown under drought stress. Phytochemistry. 2009;70(9):1107-16. https://doi.org/10. 1016/j.phytochem.2009.07.008.

16. D'Amelia V, Aversano R, Ruggiero A, Batelli G, Appelhagen I, Dinacci C, Hill L, Martin C, Carputo D. Subfunctionalization of duplicate MYB genes in Solanum commersonii generated the cold-induced ScAN2 and the anthocyanin regulator ScAN1. Plant Cell Environ. 2018;41(5):1038-51. https:// doi.org/10.1111/pce.12966

17. Liu Y, Wang L, Zhang J, Yu B, Wang J, Wang D. The MYB transcription factor StMYBA1 from potato requires light to activate anthocyanin biosynthesis in transgenic tobacco. J Plant Biol. 2017;60(1):93-101. https://doi.org/10.1007/ s12374-016-0199-9.

18. Montefiori M, Brendolise C, Dare AP, Lin-Wang K, Davies KM, Hellens RP, Allan AC. In the Solanaceae, a hierarchy of bHLHs confer distinct target specificity to the anthocyanin regulatory complex. J Exp Bot. 2015;66(5): 1427-36. https://doi.org/10.1093/jxb/eru494.

19. Chorev M, Carmel L. The function of introns. Front Genet. 2012;3. https:// doi.org/10.3389/fgene.2012.00055.

20. Jo BS, Choi SS. Introns: the functional benefits of introns in genomes. Genomics Inform. 2015;13(4):112. https://doi.org/10.5808/Gl.2015.13.4.112.

21. Liu Y, Tikunov Y, Schouten RE, Marcelis LF, Visser RG, Bovy A. Anthocyanin biosynthesis and degradation mechanisms in Solanaceous vegetables: a review. Front Chem. 2018;6:52. https://doi.org/10.3389/fchem.2018.00052.

22. Liu Y, Lin-Wang K, Deng C, Warran B, Wang L, Yu B, Yang H, Wang D, Espley RV, Zhang J, Wang D, Allan AC. Comparative transcriptome analysis of white and purple potato to identify genes involved in anthocyanin biosynthesis. PLoS One. 2015;10(6):e0129148. https://doi.org/10.1371/journal.pone. 0129148.

23. Zong Y, Xi X, Li S, Chen W, Zhang B, Liu D, Liu B, Wang D, Zhang H. Allelic variation and transcriptional isoforms of wheat TaMYC1 gene regulating anthocyanin synthesis in pericarp. Front Plant Sci. 2017;8:1645. https://doi. org/10.3389/fpls.2017.01645.

24. Jiang W, Liu T, Nan W, Jeewani DC, Niu Y, Li C, Wang Y, Shi X, Wang C, Wang J, Li Y, Gao X, Wang Z. Two transcription factors TaPpm1 and TaPpb1 co-regulate anthocyanin biosynthesis in purple pericarps of wheat. J Exp Bot. 2018;69(10):2555-67. https://doi.org/10.1093/jxb/ery101. 